J. Sustain. Wireless Syst.

Vol.02/ No. 2

Pages: 59-67

http://irojournals.com/irosws/

DOI: https://doi.org/10.36548/jsws.2020.2.001

\title{
WSN based Improved Bayesian Algorithm Combined with Enhanced Least-Squares Algorithm for Target Localizing and Tracking
}

Dr. Wang Haoxiang,

Director and lead executive faculty member,

GoPerception Laboratory,

NY, USA

Email id: hw496@goperception.com.

Dr. S. Smys,

Professor,

Department of CSE,

RVS Technical Campus,

Coimbatore, India.

Email id: smys375@gmail.com

Abstract: For wireless sensor network (WSN), localization and tracking of targets are implemented extensively by means of traditional tracking algorithms like classical least-square (CLS) algorithm, extended Kalman filter (EKF) and the Bayesian algorithm. For the purpose of tracking and moving target localization of WSN, this paper proposes an improved Bayesian algorithm that combines the principles of least-square algorithm. For forming a matrix of range joint probability and using target predictive location of obtaining a sub-range probability set, an improved Bayesian algorithm is implemented. During the dormant state of the WSN testbed, an automatic update of the range joint probability matrix occurs. Further, the range probability matrix is used for the calculation and normalization of the weight of every individual measurement. Lastly, based on the weighted least-square algorithm, calculation of the target prediction position and its correction value is performed. The accuracy of positioning of the proposed algorithm is improved when compared to variational Bayes expectation maximization (VBEM), dual-factor enhanced VBAKF (EVBAKF), variational Bayesian adaptive Kalman filtering (VBAKF), the fingerprint Kalman filter (FKF), the position Kalman filter (PKF), the weighted K-nearest neighbor (WKNN) and the EKF algorithms with the values of $0.5 \%, 7 \%, 14 \%, 19 \%, 33 \%$ and $35 \%$ respectively. Along with this, when compared to Bayesian algorithm, the computation burden is reduced by the proposed algorithm by a factor of over $80 \%$.

Keywords: Prediction position; Improved Bayesian enhanced Least-Squares; Localization and tracking; Wireless sensor networks;

\section{Introduction}

In applications relating to military surveillance, traffic control, moving target tracking, navigation for blind person, border monitoring and so on, wireless sensor networks (WSNs) are used widely [1]. In case of military surveillance, mobile tracking technology is implemented whereas for localizing and tracking the targets in air and ground, the use of WSN application for moving target tracking is implemented. Long-term work, easy deployment and low cost are the major benefits of WSN [2]. However, in applications that involve positioning and localization, several challenges in the 
J. Sustain. Wireless Syst.

Vol.02/ No. 2

Pages: 59-67

http://irojournals.com/irosws/

DOI: https://doi.org/10.36548/jsws.2020.2.001

implementation of sensor nodes with constraints in the distance of communication, random distribution, large scale, poor reliability and limited energy occurs [3].

In WSN, tracking of manoeuvring targets is challenging. Tracking algorithm significantly affects the computation time and accuracy of the system in localizing and tracking of the target [4]. The traditional algorithms like CLS algorithm, Kalman filter, fuzzy comprehensive function based track association algorithm, Bayesian algorithm and so on are used for performing tracking [5]. Mobile sensor network based pursuit and localization of multiple target using energy-based tracking and localization techniques are also in use along with velocity estimation using vision based position and nonholonomic mobile robots for adaptive trajectory tracking, in case of information that is highly incompetent, subspaces tracking and online identification, positioning system design using GDOP analysis, real-time compressive tracking and wireless sensor network applications based on cameras with mechanisms for efficient tracking using cluster-based techniques [6]. For evaluating the algorithm performance, we use some of these techniques in this paper.

Errors occur in code phrases and range measurement with effects of signal shadowing and multipath in the sensor nodes due to the noise in the receiver. Position estimation errors occur due to the errors in pseudo range measurement [7]. At various levels, the WSN target tracking results are influenced due to the sensor noise and disturbance in the real time scenario. The target information can be reflected accurately by the WSN by means of the sensory information that has been gathered. However, certain researchers have considered the sensory information errors as well. In WSN target tracking the accuracy can be affected by the sensory data error by a great extent. Use of probability matrix for analyzing the measurements for each cycle and aggregation of complex calculation for reducing the time consumption of tracking algorithm can also be performed [8]. This aids updating the matrix during the dormant mode of the system and overcoming the need to execute complex functions at every individual cycle. Based on this ides, complex computation along with tracking algorithm can be used for improving the realtime system performance. With limited computational performance and energy, the positioning requirements of WSN is met with the algorithm implemented in this paper by reducing the sensor measurement information error [9].

\section{Related Works}

In linear state equations, the target maneuvering is more efficient with the use of $\mathrm{KF}$ frameworks [10,11]. Accurate performance for tracking cannot be guaranteed by algorithms for non-gaussian noise and nonlinear maneuvering for tracking of targets. A unified and specific data can be produced by using information from multiple sources and integrating by means of a process called multi-sensor data fusion (MSDF) technique [12]. This process aids in obtaining information that is meaningful, efficient and accurate and cannot be detected with a single sensor. Accurate estimation cannot be achieved by EKF sue to the nonlinear maneuvering target of the measurement model. Combination of soft computing 
J. Sustain. Wireless Syst.

Vol.02/ No. 2

Pages: 59-67

http://irojournals.com/irosws/

DOI: https://doi.org/10.36548/jsws.2020.2.001

technology and EKF is a major topic of research for achieving highly accurate estimation. However, the work by combining these approaches has been done by very few researchers [13].

In the sense of minimum mean squared error, there is an optimal KF-based framework for Gaussian systems [14]. It is a challenging issue to estimate the parameter states in case of non-Gaussian and nonlinear systems. For nominal attribute classification issues, efficient implementation of naive Bayesian classifiers (NBC) can be done. Two handling strategies namely destiny estimation and discretization are used in general for tasks that have continuous attributes [15].

\section{Proposed Work}

The proposed algorithm makes use of improved Bayesian algorithm combined with enhanced leastsquares algorithm for analysis of the target measurements that are gathered by the WSN sensor nodes. Cartesian coordinate technique is used for determination of position and prediction of the position of the target. Position determination and position prediction analysis are the major tasks to be performed in this section. In WSNs, during occurrence of uncertain events like change of motion, slowing down or acceleration of moving target, the tracking process is challenging [16]. Loss of target or false target may occur if matching of actual moving model and the target maneuver model is not performed. Constant acceleration (positive acceleration), constant deceleration (negative acceleration) or uniform motion ( 0 acceleration) is similar to the target motion model when the two adjacent measurements have minimal time interval [17]. Associating the state equation to the two adjacent measurements provides the following expression:

$$
\begin{gathered}
A_{k}=\tilde{X} \cdot A_{k+1}+\hat{X} \cdot \omega \text {---- }(1) \\
A_{k}=\left[a_{k}, a_{k}^{\prime}, a_{k}^{\prime \prime}, b_{k}, b_{k}^{\prime}, b_{k}^{\prime \prime}\right]^{X} \text {---- (2) } \\
\tilde{X}=\left[\begin{array}{cccccc}
1 & x & \frac{1}{2} x^{2} & 0 & 0 & 0 \\
0 & 1 & x & 0 & 0 & 0 \\
0 & 0 & 1 & 0 & 0 & 0 \\
0 & 0 & 0 & 1 & x & \frac{1}{2} x^{2} \\
0 & 0 & 0 & 0 & 1 & x \\
0 & 0 & 0 & 0 & 0 & 1
\end{array}\right]-(3) \\
\hat{X}=\left[\begin{array}{cccccc}
\frac{x^{2}}{2} & x & 1 & 0 & 0 & 0 \\
0 & 0 & 0 & \frac{x^{2}}{2} & x & 1
\end{array}\right]---(4), \omega=\left[\begin{array}{l}
\omega_{a} \\
\omega_{b}
\end{array}\right]
\end{gathered}
$$


J. Sustain. Wireless Syst.

Vol.02/ No. 2

Pages: 59-67

http://irojournals.com/irosws/

DOI: https://doi.org/10.36548/jsws.2020.2.001

Here, the target's state vector is $A_{k} \in R^{6 \times 6}$. Along the $x$-axis, the position is given by $a_{k}$, velocity is given by $\mathrm{a}_{\mathrm{k}}{ }_{\mathrm{k}}$ and the moving target acceleration is represented as $\mathrm{a}^{\prime \prime}{ }_{\mathrm{k}}$. Similarly, along the y-axis, the term $b_{\mathrm{k}}$ represents the position, $b^{\prime}{ }_{k}$ represents the velocity and $b^{\prime \prime}{ }_{k}$ represents the moving target acceleration. The sampling period is given by $x$. Among with zero meaning Gauss white noise, $\omega_{\mathrm{a}}$ and $\omega_{\mathrm{b}}$ are autonomous of each other. Their respective variances are given by $\sigma_{\omega \mathrm{a}}$ and $\sigma_{\omega \mathrm{\omega b}}$. The state vector equation of the target can hence be modified as follows:

$$
A_{k}=F_{k, k-1} A_{k-1}+W_{k-1}------(6)
$$

A mathematical model is essential for the detection system for localization and tracking of the target. In this model, the measured sensor values, the sensor position and the targets predicted position are included. Based on the geometry of the relationship between the position measured and the position predicted in the nodes, calculation of the deviation between the measured value and predicted position is performed. The target measurement position and the sensor i position as well as the predicted target position at $\mathrm{k}$ time is estimated and their relationship is established geometrically. At time $\mathrm{k}$, the target position predicted is given by $a_{k}$ and $b_{k}$. The sensor coordinates are represented by $a_{s i}$ and $b_{s i}$. The sensor position can be estimated using the following expression for the required target:

$$
\left\{\begin{array}{l}
\Delta a_{k i}=a_{k}-a_{k i}=\sigma_{i}^{\prime} \sin \left(\theta^{\prime}{ }_{i}\right)-\sigma_{i} \sin \left(\theta_{i}\right) \\
\Delta b_{k i}=b_{k}-b_{k i}=\sigma_{i}^{\prime} \cos \left(\theta^{\prime}{ }_{i}\right)-\sigma_{i} \cos \left(\theta_{i}\right)
\end{array}\right.
$$

\section{Results and Discussion}

The experimental specifications of WSN testbed is provided in this section. The battery capacity, storage capacity and processor performance that has similar measurement function are enhanced using the WSN testbed with the sink node. Data transmission is performed using wireless module for facilitating the sensor nodes deployment. At every cycle, around $3 \mathrm{~Kb}$ or 50 groups of information is to be transmitted by a node based on infrared ranging module sampling frequency. The data is labeled with the sensor count, distance between the target and the node as well as the direction angle that is available for all the data groups. Corresponding to the number of every node, the precise position is stored by the sink node. Calculation of the measured position can be performed by the accurate sensor position corresponding to the distance between the target and the node, direction angle and the number of the sink node. Further, the calculated results are transmitted by the sink node to the computer. 
J. Sustain. Wireless Syst.

Vol.02/ No. 2

Pages: 59-67

http://irojournals.com/irosws/

DOI: https://doi.org/10.36548/jsws.2020.2.001

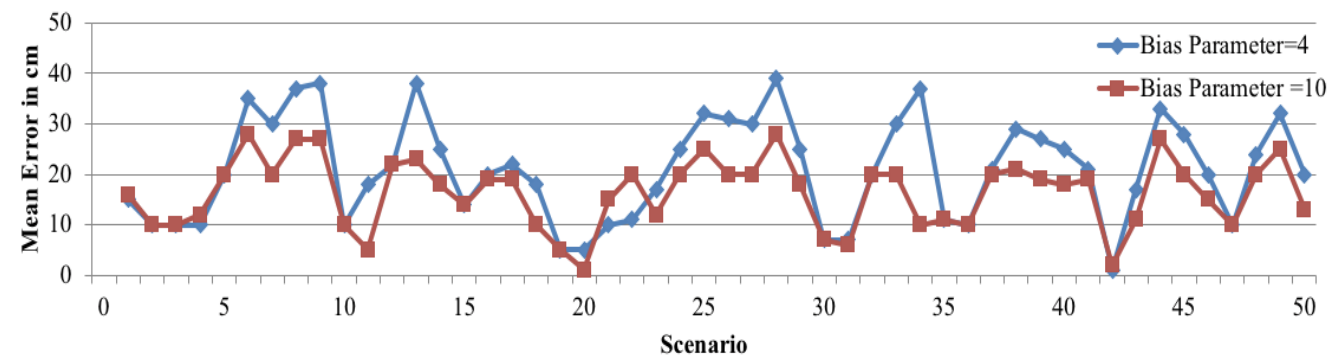

(a)

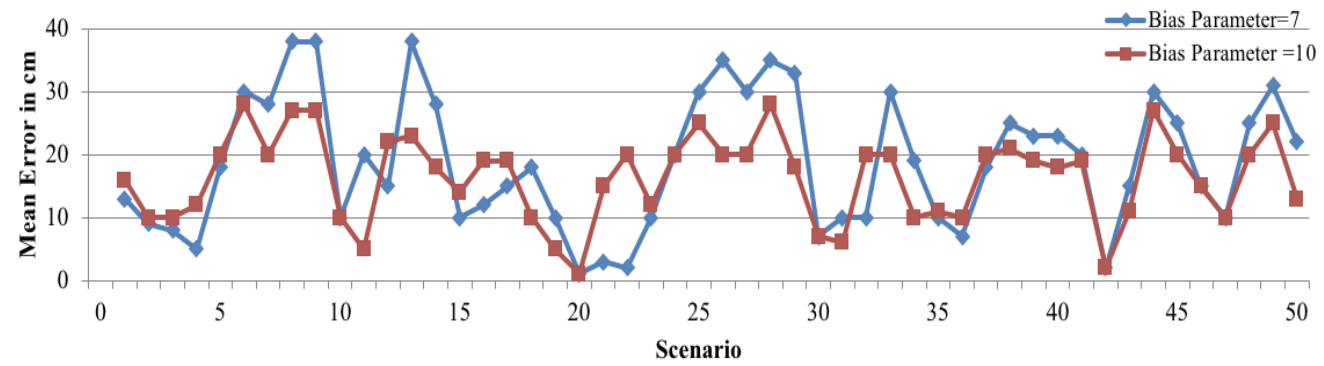

(b)

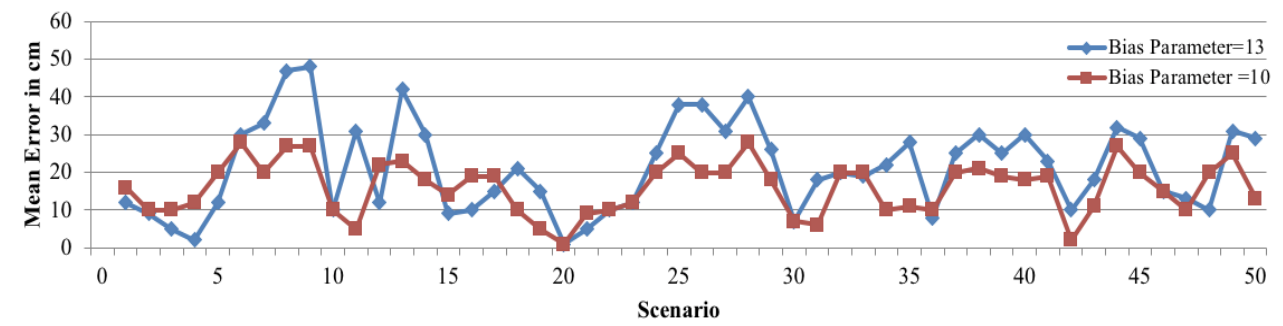

(c)

Figure 1: Mean error comparison for various bias parameters

The proposed algorithm is estimated for four different bias parameters and the mean error for these values is compared as represented in figure 1 . When compared to the bias values of 13,7 and 4 , the mean error is smaller to the value 10. Using the proposed algorithm, we also perform a comparison for four different bias parameter values of the moving target time consumption. The time consumption is almost similar for all the four values chosen. Further, the results are compared for the range of parameters between 1 to 16 . It is found that the value of 10 is obtained for mean error which is the smallest of the entire range.

Table 1: Comparison of performance for various bias parameters

\begin{tabular}{|c|c|c|c|}
\hline Bias parameter & Mean Error & Bias parameter & Mean Error \\
\hline 9 & 14.69 & 1 & 23.94 \\
\hline 10 & 14.53 & 2 & 21.75 \\
\hline
\end{tabular}


J. Sustain. Wireless Syst.

Vol.02/ No. 2

Pages: 59-67

http://irojournals.com/irosws/

DOI: https://doi.org/10.36548/jsws.2020.2.001

\begin{tabular}{|l|l|l|l|}
\hline 11 & 14.55 & 3 & 20.08 \\
\hline 12 & 16.44 & 4 & 18.99 \\
\hline 13 & 16.01 & 5 & 17.74 \\
\hline 14 & 15.16 & 6 & 16.75 \\
\hline 15 & 15.07 & 7 & 15.73 \\
\hline 16 & 16.15 & 8 & 15.31 \\
\hline
\end{tabular}

In the experimental setup, the bias parameter is set to be 10 . The implementation of proposed algorithm and its calculated results are represented along with the standard deviation in table 1 based on special bias parameters.

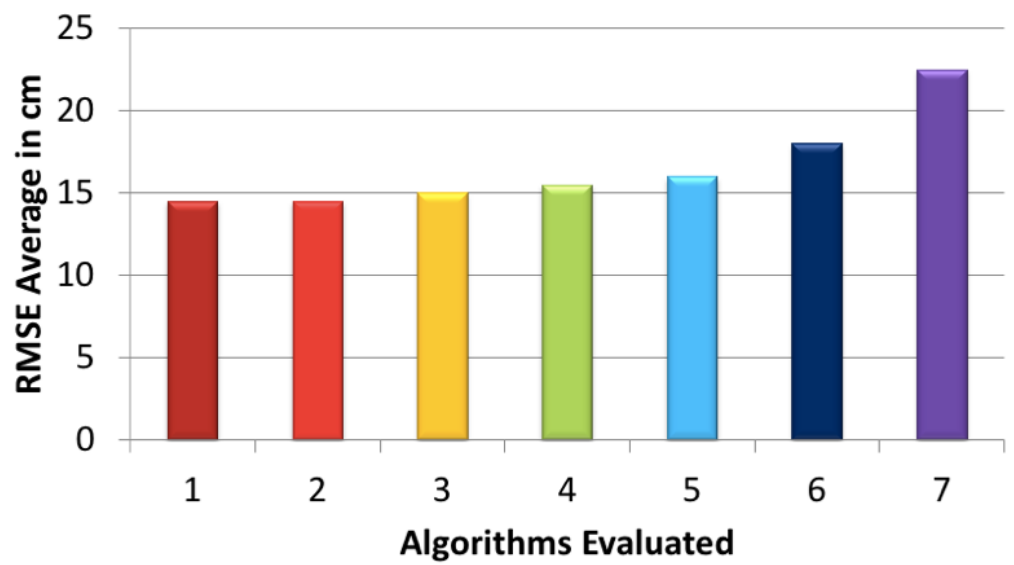

Figure 2: Comparison of proposed algorithm, VBEM, dual-factor EVBAKF, VBAKF, FKF, PKF and WKNN in terms of average RMSE value

Despite the occurrence of unobservable variables, the complex posterior distribution can be calculated and approximated using variational Bayesian inference. The variational Bayesian algorithm and its performance is affected by the approximation results and their randomness. Prior to training, when compared to the variational Bayesian inference, the proposed algorithm offers better approximate of the calculated posterior distribution. In comparison to the other algorithms, a stable and higher positioning precision is offered by the proposed algorithm. Figure 2 provides the comparison of proposed algorithm, VBEM, dual-factor EVBAKF, VBAKF, FKF, PKF and WKNN in terms of average RMSE value. 
J. Sustain. Wireless Syst.

Vol.02/ No. 2

Pages: 59-67

http://irojournals.com/irosws/

DOI: https://doi.org/10.36548/jsws.2020.2.001

\section{Conclusion}

In this paper, localization and tracking of moving targets is done in WSN using improved Bayesian algorithm. A range probability matrix is formed and the target predictive location is used for obtaining a sub-range probability set by means of an improved Bayesian algorithm. When the testbed of WSN is in sleep state, automatic updating of the region probability matrix is performed. Deletion of false measurement information is done on calculation of the individual measurement data's true probability on the basis of region joint probability matrix. Calculation of true probability information is performed and optimization of the effective measurement information weight values is done. Further, weighted least-squares algorithm is implemented with optimized weights. WSN testbed is used for validation of the proposed algorithm using experiments. In comparison to the EKF-CMAC, the localization RMSE can be reduced using the proposed algorithm by a factor of over $35 \%$ using the proposed algorithm. In comparison to the Bayesian algorithm, the consumption of computational time is reduced using the proposed algorithm. The trajectory estimation algorithm based on WSN and its performance is too optimized in the future work.

\section{References}

[1] Yetgin, H., Cheung, K. T. K., El-Hajjar, M., \& Hanzo, L. H. (2017). A survey of network lifetime maximization techniques in wireless sensor networks. IEEE Communications Surveys \& Tutorials, 19(2), 828-854.

[2] Haoxiang, W., \& Smys, S. (2020). Soft Computing Strategies for Optimized Route Selection in Wireless Sensor Network. Journal of Soft Computing Paradigm (JSCP), 2(01), $1-12$.

[3] Raj, J. S. (2020). Machine Learning Based Resourceful Clustering With Load Optimization for Wireless Sensor Networks. Journal of Ubiquitous Computing and Communication Technologies (UCCT), 2(01), 29-38.

[4] Ranganathan, G., \& Smys, S. Smart Wireless sensors for Impairment detection of the offshore Wind Turbines.

[5] Muneera Begum H, D. A. Janeera, and AG, Aneesh Kumar. "Internet of Things based Wild Animal Infringement Identification, Diversion and Alert System", Fifth International Conference on Inventive Computation Technologies (ICICT-2020), pp. 672-676. IEEE, 2020.

[6] Lin, S., Miao, F., Zhang, J., Zhou, G., Gu, L., He, T., ... \& Pappas, G. J. (2016). ATPC: adaptive transmission power control for wireless sensor networks. ACM Transactions on Sensor Networks (TOSN), 12(1), 1-31.

[7] Biswas, S., Das, R., \& Chatterjee, P. (2018). Energy-efficient connected target coverage in multi-hop wireless sensor networks. In Industry interactive innovations in science, engineering and technology (pp. 411-421). Springer, Singapore.

[8] He, X., Wang, T., Liu, W., \& Luo, T. (2019). Measurement data fusion based on optimized weighted least-squares algorithm for multi-target tracking. IEEE Access, 7, 13901-13916. 
J. Sustain. Wireless Syst.

Vol.02/ No. 2

Pages: 59-67

http://irojournals.com/irosws/

DOI: https://doi.org/10.36548/jsws.2020.2.001

[9] Tomic, S., Beko, M., Dinis, R., Tuba, M., \& Bacanin, N. (2017). Bayesian methodology for target tracking using combined RSS and AoA measurements. Physical Communication, 25, 158-166.

[10] Cheng, L., Feng, L., \& Wang, Y. (2018). A residual analysis-based improved particle filter in mobile localization for wireless sensor networks. Sensors, 18(9), 2945.

[11] Rana, M. M., \& Shuvo, M. M. R. (2018, December). Localization of Senor Nodes Using Weighted Least Squares Algorithm: Comprehensive Literature Review and Future Research Directions. In 2018 International Conference on Innovation in Engineering and Technology (ICIET) (pp. 1-6). IEEE.

[12] Tomic, S., Beko, M., Dinis, R., \& Bernardo, L. (2018). On target localization using combined RSS and AoA measurements. Sensors, 18(4), 1266.

[13] Kumar, S., \& Hegde, R. M. (2017). A review of localization and tracking algorithms in wireless sensor networks. arXiv preprint arXiv:1701.02080.

[14] Cheng, L., Li, Y., Wang, Y., Bi, Y., Feng, L., \& Xue, M. (2019). A triple-filter NLOS localization algorithm based on fuzzy c-means for wireless sensor networks. Sensors, 19(5), 1215.

[15] Kumar, D. P., Amgoth, T., \& Annavarapu, C. S. R. (2019). Machine learning algorithms for wireless sensor networks: A survey. Information Fusion, 49, 1-25.

[16] Jondhale, S. R., Shubair, R., Labade, R. P., Lloret, J., \& Gunjal, P. R. (2020). Application of Supervised Learning Approach for Target Localization in Wireless Sensor Network. In Handbook of Wireless Sensor Networks: Issues and Challenges in Current Scenario's (pp. 493-519). Springer, Cham.

[17] Gumaida, B. F., \& Luo, J. (2019). A hybrid particle swarm optimization with a variable neighborhood search for the localization enhancement in wireless sensor networks. Applied Intelligence, 49(10), 3539-3557.

\section{Authors Biography}

Dr. Haoxiang Wang is currently the director and lead executive faculty member of GoPerception Laboratory, NY, USA . His research interests include multimedia information processing, pattern recognition and machine learning, remote sensing image processing and data-driven business intelligence. He has co-authored over 60 journal and conference papers in these fields on journals such as Springer MTAP, Cluster Computing, SIVP; IEEE TII, Communications Magazine; Elsevier Computers \& Electrical Engineering, Computers, Environment and Urban Systems, Optik, Sustainable Computing: Informatics and Systems, Journal of Computational Science, Pattern Recognition Letters, Information Sciences, Computers in Industry, Future Generation Computer Systems; Taylor \& Francis International Journal of Computers and Applications and conference such as IEEE SMC, ICPR, ICTAI, ICICI, CCIS, ICACI 
J. Sustain. Wireless Syst.

Vol.02/ No. 2

Pages: 59-67

http://irojournals.com/irosws/

DOI: https://doi.org/10.36548/jsws.2020.2.001

Dr. S. Smys received his M.E and Ph.D degrees all in Wireless Communication and Networking from Anna University and Karunya University, India. His main area of research activity is localization and routing architecture in wireless networks. He serves as Associate Editor of Computers and Electrical Engineering (C\&EE) Journal, Elsevier and Guest Editor of MONET Journal, Springer. He is served as a reviewer for IET, Springer, Inderscience and Elsevier journals. He has published many research articles in refereed journals and IEEE conferences. He has been the General chair, Session Chair, TPC Chair and Panelist in several conferences. He is member of IEEE and senior member of IACSIT wireless research group. He has been serving as Organizing Chair and Program Chair of several International conferences, and in the Program Committees of several International conferences. Currently 